\title{
Pertussis: novas estratégias de vacinação para prevenção de uma antiga doença
}

\section{Pertussis: new vaccination strategies to prevent an old disease}

\author{
Lucia Ferro Bricks ${ }^{1}$ \\ 1. Pediatra e Doutora em Medicina pela FMUSP. Diretora de Saúde Pública da Sanofi Pasteur, Divisão Vacinas da Sanofi, Brasil.
}

\begin{abstract}
Resumo
Introdução: A despeito das elevadas coberturas vacinais, a pertussis persiste como importante causa de hospitalizações e mortes em lactentes, sendo necessárias novas estratégias de vacinação para o controle da doença. Objetivos: Descrever as recentes mudanças na epidemiologia da coqueluche, analisar as principais fontes de transmissão para lactentes, os fatores envolvidos no ressurgimento da doença e as publicações sobre uso de vacinas acelulares em adolescentes e adultos. Métodos: Foram identificados e analisados os artigos publicados no PUBMED e SCIELO, assim como as informações disponíveis nos sites oficiais da OMS, OPAS, CDC, ECDC e do Ministério da Saúde do Brasil, de 01/01/2003 a 31/12/2012. Conclusões: A reemergência da pertussis foi constatada em países que adotam diferentes vacinas e esquemas de imunização. As mais relevantes fontes de pertussis para lactentes, o grupo de mais alto risco para complicações e mortes, são os contatos domiciliares, especialmente, as mães. A proteção oferecida por infecção natural ou por vacinas é limitada. As vacinas Tdap e Tdap-IPV são seguras e imunogênicas, e dados limitados demonstraram que essas vacinas conferem proteção direta e indireta, com efetividade de, aproximadamente $80 \%$, durante 5 anos. É necessário melhorar a educação sobre os benefícios da vacinação de adolescentes e adultos e aprimorar a vigilância da pertussis nesses grupos com maior utilização novos recursos laboratoriais (PCR e sorologia) a fim de identificar o intervalo ideal para administrar as doses de reforço e as melhores estratégias de vacinação para reduzir o número de mortes em lactentes.
\end{abstract}

Palavras-chave: Pertussis. Coqueluche. Incidência. Diagnóstico. Transmissão. Fatores de virulência. Vacina contra coqueluche. Imunização.

\begin{abstract}
Background: Despite high childhood immunization coverage rates, pertussis remains an important cause of hospitalization and deaths in infants, and new strategies of vaccination are necessary to control this serious disease. Objectives: To describe recent changes in pertussis epidemiology, to analyse the most frequent sources of infection to young babies, the most relevant factors involved in the reemergency of the disease and to make a critical review of recent information data about acellular vaccines recommended as booster for adolescents and adults. Methods: Articles were retrieved from PUBMED, SCIELO and oficial sites of WHO, PAHO, CDC, ECDC and Brazil's Ministry of Health, from 2003/01/01 to 2012/12/31. Conclusions: The reemergency of pertussis was identified in countries that adopted different vaccines and schedules of immunization. The most relevant sources of pertussis to infants, the most important group at risk for complications and deaths, are close contacts, especially the mothers. The protection offered by natural infection and vaccines is limited. Tdap and Tdap-IPV are immunogenic and safe, and limited data showed that the effectiveness is about $80 \%$ along 5 years. Vaccination of adolescents and adults can offer direct and indirect protection (herd immunity). It is necessary more education about the benefits of these vaccines for adolescents and adults to get better acceptation of new strategies of vaccination, and to improve the pertussis surveillance among these groups with the introduction of new lab tests (PCR and serology) in order to find the ideal interval for the administration of the boosters and the best strategies to avoid pertussis deaths in infants.
\end{abstract}

Key-words: Bordetella pertussis. Whooping cough. Incidence. Diagnosis. Transmission. Virulence Factors, Bordetella. Pertussis Vaccine. Immunization.

\section{INTRODUÇÃO}

Pertussis (coqueluche ou tosse comprida) é uma doença respiratória causada pela bactéria Bordetella pertussis, que tem distribuição global e ocorre de forma endêmica, com ciclos epidêmicos a cada 2 a $5 \operatorname{anos}^{1-3}$. A incidência da coqueluche apresenta grandes variações em países de uma mesma região e em diferentes regiões de um mesmo país ${ }^{3-23}$.

Apesar de existirem vacinas para a prevenção da coqueluche desde o final da década de $1940^{24-26}$ e de as coberturas vacinais no primeiro ano de vida terem aumentado substancialmente nas duas últimas décadas ${ }^{1,2}$, de acordo com a OMS, o número de casos e de mortes por coqueluche, em 2008, foi superior a 16 milhões e 195.000, respectivamente. O fato de terem sido notificados à OMS apenas 139.382 casos de coqueluche em 2010 demonstra claramente que a doença é subdiagnosticada. ${ }^{1}$

Embora a coqueluche doença acometa pessoas de qualquer faixa etária, a maioria dos casos e quase todas as

Correspondência: lfbricks@gmail.com

Conflito de interesse: Há conflito de interesses. Diretora de Saúde Pública da Sanofi Pasteur, Divisão de Vacinas da Sanofi no Brasil.

Recebido em 08 Jan 2013; Revisado em 16 Abr 2013; Aceito em 16 Abr 2013 
mortes são registradas em bebês menores de seis meses. Paradoxalmente, as taxas de incidência e o número de mortes por coqueluche aumentaram de forma substancial nos países onde as coberturas vacinais são elevadas, sendo necessário rever os fatores associados com a reemergência da doença e as estratégias para sua prevenção ${ }^{3-26}$.

O objetivo deste artigo é apresentar uma revisão crítica da literatura sobre os aspectos epidemiológicos e os fatores associados com a reemergência da coqueluche e as novas estratégias para prevenir a doença.

Os artigos foram identificados através de pesquisa nas bases de dados do PubMed e SciELO, assim como nos sites da World Health Organization (WHO), Pan American Health Organization (PAHO), Centers for Disease Control and Prevention (CDC), European Centre for Disease Prevention and Control (ECDC), do Ministério da Saúde do Brasil, no período de 01/01/2003 a 31/12/2012. Foram levantados os artigos publicados em inglês, português, espanhol e francês, utilizando-se as seguintes palavras-chave: pertussis, incidência, diagnóstico, transmissão, vacinas, imunização.

\section{ASPECTOS EPIDEMIOLÓGICOS E CLÍNICOS DA PERTUSSIS}

As taxas de incidência da coqueluche são muito variáveis s,13,16,20 $^{\mathrm{e}}$, nos últimos anos, aumentaram em diversos países com altas coberturas vacinais, incluindo o Brasil ${ }^{1,3-23}$. Nos EUA, o aumento nas taxas de incidência foi alarmante, tendo sido registrados mais de 20.000 casos da doença em 2004, 2005, 2010 e 2012, com epidemias em diversos estados ${ }^{3,7-9}$. Na Austrália, em 2011, foram registrados mais 38.040 casos da doença ${ }^{1,11}$. Na Europa, os mais altos coeficientes de incidência têm sido registrados na Holanda, Suíça, Noruega ${ }^{13}$; entretanto, significativo aumento no número de casos também tem sido observado em algumas regiões da Espanha ${ }^{15}$ e Inglaterra ${ }^{14}$. No Reino Unido, em 2012, foram confirmados 8.819 casos de pertussis e, apenas no mês de outubro, 1.631 casos, com 10 mortes em lactentes jovens ${ }^{14}$.

Tanto nos EUA como na Europa, observou-se desvio na faixa etária, com substancial aumento no número de casos de pertussis em adolescentes e adultos. ${ }^{16}$ Apesar disso, os maiores coeficientes de incidência e quase todas as mortes são registradas em bebês com menos de seis meses de idade. .,4,7-15,17-23 $^{-2}$

Diversos fatores podem estar associados à reemergência da coqueluche $3,4,7-10,14,16-34$ :

1) a proteção conferida pela infecção natural, ao contrário do que muitos acreditam, não é permanente, e se reduz, quando existe redução da circulação da bactéria na comunidade (redução do booster natural);

2) a eficácia das melhores vacinas contra a coqueluche é de, aproximadamente, $80 \%$, e o acúmulo de suscetíveis predispõe a epidemias;
3) a introdução de métodos diagnósticos mais sensíveis e específicos, como PCR e sorologia, que permitem identificar maior número de casos, principalmente em adolescentes e adultos;

4) possível menor eficácia de vacinas acelulares, em comparação com as vacinas de células inteiras.

Estima-se que a proteção conferida por infecção natural dure entre 4 e 20 anos, e que a proteção conferida por vacinas dure entre 4 e 12 anos $^{27}$; entretanto, é preciso ter muita cautela ao analisar os dados de estudos realizados em diferentes épocas, com populações que receberam diversos tipos de vacinas, com coberturas e eficácia variáveis ${ }^{26}$. Em populações não vacinadas ou que têm baixas coberturas, existe grande circulação da $B$. pertussis na comunidade e a exposição natural à bactéria pode atuar como "booster" natural, mantendo a imunidade na população. A maioria dos países industrializados adotou as vacinas acelulares nas últimas duas décadas, após ter conseguido reduzir dramaticamente o número de casos da doença na comunidade, devido às altas coberturas vacinais. Estima-se que $90 \%$ das mortes por coqueluche ainda ocorram em países em desenvolvimento, onde as coberturas vacinais são baixas, tanto no esquema básico, como nas doses de reforço².

A proteção conferida pelas melhores vacinas acelulares é semelhante à observada após o uso das melhores vacinas de células inteiras, que têm eficácia de, aproximadamente, $80 \% .^{2}$ O acúmulo de suscetíveis devido a falhas primárias (não indução de resposta imune apropriada) ou secundárias (redução nos títulos de anticorpos protetores) das vacinas propicia a maior circulação da $B$. pertussis e a ocorrência de epidemias, que ocorrem a cada 2 a 5 anos, mesmo em locais onde as coberturas vacinais na rotina são superiores a 95\% ${ }^{24-33}$. Embora alguns autores associem a reemergência da coqueluche à menor efetividade das vacinas acelulares em comparação com as vacinas de células inteiras, nos EUA, a menor incidência de pertussis foi registrada em 1976, aumentando a partir da década de 80 , antes da introdução das vacinas acelulares na rotina ${ }^{27-30}$. Além disso, a reemergência da coqueluche também tem sido registrada em países que utilizam vacinas de células inteiras na rotina, como Costa Rica ${ }^{17}$, Panamá ${ }^{18}$, Uruguai ${ }^{19}$, Argentina $^{21}$, Colômbia ${ }^{22}$, Chile $^{23}$ e Brasil $^{4,5}$.

No Brasil, apesar dos baixos coeficientes de incidência da pertussis, o número de casos e mortes por coqueluche aumentou significativamente nos dois últimos anos ${ }^{1,4}$. Em 2011, foram notificados 2.258 casos e 55 mortes $^{5}$ e, de janeiro a dezembro de 2012, 5.124 casos e 86 mortes, a maioria bebês menores seis meses de idade ${ }^{5}$.

O único reservatório da $B$. pertussis é o ser humano ${ }^{3,24-27}$. A bactéria é transmitida de pessoa a pessoa, por meio de gotículas respiratórias e, como tem baixa sobrevida no meio ambiente, geralmente é adquirida através do contato próximo com indivíduo contaminado, que pode, ou não, apresentar manifestações clínicas ${ }^{24-33}$. 
Após um período de incubação de 9 a 10 dias (faixa 6 a 20 dias), a doença se inicia com sintomas inespecíficos (coriza, tosse e febre). Nessa fase, conhecida como fase catarral, o risco de transmissão é muito alto e a taxa de ataque secundário para contatos domiciliares não imunes é de, aproximadamente, $90 \%{ }^{2}$. Estima-se que em população não imune um caso primário gere, em média, 15 a 17 novos casos $\left(R_{0}=15\right.$ a 17$) .{ }^{27,30}$ Pessoas não tratadas podem excretar a bactéria por três semanas. O risco de transmissão da $B$. pertussis é baixo na fase paroxística e praticamente inexistente cinco dias após a introdução de quimoprofilaxia com antibióticos apropriados (macrolídeos) ${ }^{2,3}$.

Após uma a duas semanas, a tosse piora, ocorrendo em surtos com 5 a 10 tossidas seguidas, que levam à eliminação do ar dos pulmões e, frequentemente, são seguidas por um estridor inspiratório (guincho ou whooping), vômitos e esgotamento físico. Essa fase, conhecida como paroxística, dura uma a duas semanas, é mais comumente observada em lactentes jovens, que frequentemente apresentam crises de cianose e apneia. A coqueluche é considerada doença grave em lactentes jovens, especialmente nos que ainda não receberam nenhuma dose da vacina. De acordo com o CDC, $57 \%$ das crianças menores de um ano que adquirem coqueluche são hospitalizadas devido às complicações que, geralmente ocorrem na fase paroxística. As complicações mais comuns em lactentes são: crises de apneia (67\%), pneumonia (23\%), convulsão (1\%) e encefalopatia $(0,4 \%)$. A taxa de letalidade nos EUA é de $1,6 \% .^{2}$ A fase de convalescença é prolongada, podendo durar várias semanas e as crises de tosse podem recorrer se o indivíduo apresentar outras infecções respiratórias.$^{2,3}$

A maioria das hospitalizações e mortes por coqueluche é notificada em crianças menores de um ano de idade $^{4-15,23,28-35}$. No Brasil, todas as 51 mortes por coqueluche registradas em 2012 ocorreram em menores de seis meses ${ }^{4}$ e, no Estado de São Paulo, 85\% (60 de 70) das mortes por coqueluche registradas nos últimos 10 anos ocorreram crianças menores de dois meses de idade ${ }^{34}$. Nessa faixa etária, a letalidade é alta, podendo superar 5\%. Existem evidências de que o risco de complicações e mortes por coqueluche é maior em crianças com peso de nascimento inferior a 2.500g, com escore de Apgar abaixo de 8, em filhos de mães que estudaram menos de 12 anos e em crianças que vivem em ambientes aglomerados ${ }^{31,32}$.

Em lactentes jovens, as manifestações clínicas da coqueluche podem ser indistinguíveis das causadas por outras infecções respiratórias, como a infecção por VRS, que pode ocorrer concomitantemente com a pertussis em até $30 \%$ dos $\operatorname{casos}^{36,37}$. A coinfecção com outros vírus ou bactérias parece ser comum, e pode estar associada com evolução mais grave ${ }^{38}$.

Crianças previamente vacinadas, adolescentes e adultos geralmente apresentam curso atípico da doença ${ }^{2,3,16,27-31,39-42 . ~}$ Adolescentes e adultos com coqueluche podem apresentar apenas tosse prolongada, sendo estimado que $20 \%$ ou mais dos adolescentes e adultos com tosse por mais de duas semanas apresentem pertussis ${ }^{39,41}$. A doença muitas vezes se confunde com exacerbação de quadros de asma, bronquite ou DPOC. ${ }^{43-46}$

De acordo com o CDC, menos de $5 \%$ dos adolescentes e adultos com pertussis são hospitalizados ${ }^{3}$, mas a doença está associada a alta morbidade, devido à tosse prolongada. Além disso, em adultos, a coqueluche está associada a elevadas taxas de absenteísmo à escola e/ou trabalho ${ }^{39-41}$. As complicações mais comuns no adulto são: perda de peso (33\%), perda de controle urinário (28\%), fraturas de costela (4\%) e pneumonia $(2 \%)^{3}$. Em idosos, o risco de hospitalização aumenta com a idade ${ }^{46}$.

\section{DIAGNÓSTICO}

A suspeita de coqueluche deve ser feita em todo indivíduo com tosse por mais de duas semanas, acompanhada por um ou mais dos seguintes sintomas: estridor inspiratório (guincho), tosse paroxística ou vômitos pós-tosse, independentemente da idade e antecedentes vacinais. Em lactentes jovens são comuns as crises de apnéia e, pessoas previamente vacinadas podem apresentar apenas tosse prolongada ${ }^{3,39-41}$.

Todos os casos suspeitos devem ser notificados às autoridades de saúde, e sempre que possível, deve-se colher a cultura, considerada o padrão-ouro para o diagnóstico da pertussis. Apesar de ser $100 \%$ específica, sua positividade é maior em crianças não vacinadas ou com esquema de vacinação incompleto, nas duas primeiras semanas após o início do quadro, freqüentemente antes de se levantar a suspeita diagnóstica de coqueluche. $\mathrm{Na}$ fase paroxística e após uso de antibióticos, a positividade da cultura cai significativamente, sendo comuns os resultados falsonegativos. A positividade também é baixa se o material para exame não for colhido com técnica apropriada ${ }^{3}$.

A reação em cadeia da polimerase (PCR) é um teste amplamente utilizado para o diagnóstico da coqueluche em outros países. Na Califórnia, a PCR foi introduzida em 1999 e, desde 2004, é o teste mais utilizado para confirmar coqueluche nos EUA. As vantagens da PCR são: alta sensibilidade podendo produzir resultados positivos até a quarta semana após o início da tosse, resultados rápidos e ausência de interferência após introdução de antibióticos; entretanto, esse teste ter resultados falso-positivos quando feito com apenas um marcador ou falso-negativos, se não for realizado de forma apropriada. ${ }^{3,47}$ No Brasil, a PCR raramente está disponível em serviços públicos e privados, e somente no final de 2010, foi introduzida na rede sentinela de pertussis no Estado de São Paulo ${ }^{35}$. Apesar de a introdução da PCR permitir diagnosticar maior número de casos de pertussis no estado de São Paulo, nos outros estados e em serviços que não fazem parte da rede sentinela de pertussis também foi constatado aumento significativo no número de casos, hospitalizações e mortes 
por coqueluche, indicando que o aumento da doença é real e não associado apenas à introdução da PCR. ${ }^{4,5,34,35}$

Testes sorológicos com pesquisa de IgG anti-PT (anticorpo contra toxina pertussis) são muito utilizados em países industrializados e permitiram demonstrar que adolescentes e adultos jovens apresentam infecção assintomática pela pertussis após doença natural ou vacinação ${ }^{3,48-53}$. A realização de inquéritos soroepidemiológicos em diversos países europeus permitiu identificar taxas de infecção assintomática de 1 a $6 \%$ em adolescentes e adultos jovens, demonstrando claramente que a proteção conferida por vacinas é muito limitada ${ }^{49}$.

Na Europa, os testes sorológicos são mais utilizados para diagnosticar pertussis do que nos EUA; entretanto, ainda há necessidade de padronizar os testes e critérios para sua utilização, de forma a permitir a comparação das informações sobre a doença em diferentes países. ${ }^{49,52}$ No Brasil, testes sorológicos ainda não estão disponíveis em serviços públicos ou privados, dificultando principalmente a confirmação dos casos em que suspeita diagnóstica é feita tardiamente (após quatro semanas de tosse). Altos títulos de IgG anti-PT em amostra isolada de soro indicam infecção ou vacinação recente. O pico de anticorpos geralmente ocorre 2 a 4 semanas após infecção, mas é possível fazer o diagnóstico tardio colhendo-se amostras pareadas de soro, com intervalo de 4 semanas, até 12 semanas após o início do quadro de tosse. Embora a vacinação recente possa interferir com o resultado da sorologia, seis meses após a vacinação, os títulos de anticorpos caem substancialmente, e são bem mais baixos do que os observados após infecção natural. ${ }^{53}$

\section{FONTES DE TRANSMISSÃO DA COQUELUCHE}

Nas regiões em que as coberturas vacinais são baixas, as crianças são o principal reservatório da B. pertussis, enquanto nos locais onde as coberturas vacinais na infância são altas, adolescentes e adultos jovens são as principais fontes de transmissão da bactéria ${ }^{16,24-33,54-65}$.

Em países industrializados, as principais fontes de transmissão da $B$. pertussis para lactentes jovens são os contatos domiciliares $29,31,54,57,59,62,65$.

A mãe é a principal fonte de infecção para os bebês, particularmente, para os menores de três meses de idade ${ }^{31}$. Outros contatos domiciliares como pai, irmãos e cuidadores também podem transmitir a bactéria para o bebê $\hat{0}^{55-65}$. Estudos realizados na América Latina revelaram que os avós, principalmente a avó, assim como tios, primos e outros membros da família que residem na mesma casa também têm importância na cadeia de transmissão $0^{58,60,61,64}$.

Apesar de a maioria dos estudos apontar os pais e irmãos como principais fontes de transmissão da coqueluche para lactentes jovens, em mais da metade dos casos não é possível identificar a fonte de contato entre os contatos domiciliares de lactentes jovens e estima-se que 20 e $47 \%$ dos bebês sejam infectados através de contatos casuais na comunidade ${ }^{55}$.

A maioria das investigações sobre as fontes de infecção para lactentes tem sido realizada em contatos que apresentam tosse; entretanto, a $B$. pertussis tem sido isolada das vias respiratórias de 8 a $14 \%$ dos contatos domiciliares assintomáticos de bebês que apresentam coqueluche $^{62}$. Portanto, é possível que pessoas com quadros leves ou infecção assintomática também desempenhem relevante papel na cadeia de transmissão da doença ${ }^{31,56,64,65}$.

Crianças e adolescentes previamente vacinados freqüentemente adquirem a infecção em contato com colegas de escola e amigos, enquanto os adultos adquirem coqueluche de suas crianças ou de colegas de trabalho..$^{25,26,29,30}$. Diversas epidemias de coqueluche têm sido registradas em ambientes fechados, como universidades, acampamentos, laboratórios, hospitais $2,24-26,33,39,50,57$.

Profissionais de saúde e da área são considerados de risco para adquirir a doença e também têm sido responsáveis por epidemias de coqueluche que atingem grupos de alto risco, como recém-nascidos e imunocomprometidos e, por esses motivos, esses profissionais e pessoas que têm contato com lactentes jovens (cuidadores e profissionais que trabalham em berçários e creches) também têm sido identificados como alvos prioritários para vacinação ${ }^{2,14,19-21,23,24,26,66}$.

\section{NOVAS ESTRATÉGIAS PARA PREVENIR A COQUELUCHE}

O aumento no número de casos de pertussis em adolescentes e adultos e a descoberta de que os contatos próximos de crianças são as principais fontes de infecção indicam que é necessário buscar novas estratégias de vacinação para reduzir as taxas de incidência da doença nesses grupos e para proteger, de forma indireta, os

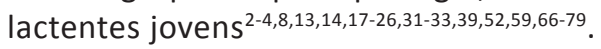

As vacinas de células inteiras são contraindicadas para pessoas maiores de 7 anos de idade ${ }^{2,24-36}$, e a vacinação de adolescentes e adultos somente tornou-se possível após o desenvolvimento de vacinas contendo menor quantidade de toxóide diftérico e de antígenos pertussis (Tdap), no final da década de $1990^{66-79}$. As vacinas Tdap contêm menor quantidade de toxóide diftérico e de antígenos da $B$. pertussis e a mesma quantidade de toxóide tetânico, em comparação com as vacinas recomendadas para a série básica de vacinação em crianças. Existem formulações em que a Tdap é combinada com a vacina inativada contra a poliomielite (Tdap-IPV), contendo as mesmas quantidades de antígenos dos vírus 
pólio 1, 2 e 3 que as vacinas formuladas para crianças e que a vacina Td-IPV, amplamente utilizada em diversos países europeus ${ }^{24,66-93}$. A vacina IPV é recomendada para adolescentes e adultos como dose de reforço em 11 países da Europa ${ }^{74}$ e para profissionais de saúde, adolescentes e adultos sem comprovação de vacinação prévia de vacinação contra a pólio, assim como para pessoas que viajam para áreas de risco para pólio ${ }^{74,80}$.

Quando houver indicação para reforço das vacinas pertussis e pólio, é preferível utilizar a vacina combinada Tdap-IPV $75,76,78,79$. Ambas as vacinas, Tdap e Tdap-IPV, podem ser utilizadas para substituir a dose de reforço com dupla-adulto (dT), pois induzem altos títulos de anticorpos contra todos os antígenos e apresentam segurança comparável às vacinas dT e dT-IPV $76,77,78,79$.

As informações sobre eficácia e duração da proteção conferida pelas vacinas Tdap e Tdap-IPV ainda são muito limitadas, não apenas devido à sua recente introdução nos EUA e Europa, como também pela baixas coberturas em adolescentes e adultos $2,24,26,66-69,75-79,81-94$.

A aceitação de vacinas é diretamente associada à conscientização sobre riscos da doença e segurança e benefícios conferidos pelas vacinas. Como a suspeita de coqueluche é feita tardiamente em adolescentes e adultos, os resultados da cultura e PCR, em geral, são negativos não sendo possível confirmar o diagnóstico nos locais onde os testes sorológicos não são disponíveis ${ }^{16,24,26}$.

Baixa percepção sobre os riscos da coqueluche tem sido associada a baixas coberturas vacinais em adolescentes e adultos e, nos EUA, Austrália e Europa, as coberturas vacinais só aumentaram significativamente depois do registro de epidemias e da exigência de vacinação para escolares e profissionais de saúde $\mathrm{e}^{7-11,13,14,91-96}$.

A OMS prioriza a vacinação de crianças, indicando a vacinação de profissionais de saúde nos países em que tiver sido confirmada a transmissão nosocomial, pois os custos para investigar a doença e introduzir profilaxia são altos ${ }^{2}$. Entretanto, deve-se ressaltar que a confirmação da transmissão da pertussis por adultos requer alto índice de suspeita e recursos laboratoriais (PCR e sorologia) raramente disponíveis em países em desenvolvimento. As autoridades de saúde de diversos países já recomendam as vacinas contra pertussis para profissionais de saúde.

Tabela 1. Recomendações de vacinas acelulares de pertussis para profissionais de saúde de acordo com instituição/País.

$\begin{array}{ll}\text { Instituição/País RECOMENDAÇÃo } & \text { RẼo }\end{array}$

$\mathrm{OMS}^{2}$

PAHO ${ }^{20}$

Estados Unidos ${ }^{84}$

Austrália ${ }^{11}$

Bélgica, Alemanha, Holanda, Luxemburgo, Reino Unido ${ }^{73}$

Áustria, Finlândia, Noruega ${ }^{73}$

França $^{73}$
Profissionais de saúde que trabalham com bebês ou imunocomprometidos, especialmente em maternidades e unidades pediátricas

Todos os profissionais de saúde que têm contato com bebês menores de 6 meses

Todos os profissionais de saúde que trabalham em hospitais ou ambulatórios e têm contato direto com o paciente

Profissionais de saúde que têm contato com bebês ou crianças

Todos os profissionais de saúde

Pediatras e profissionais de saúde em departamentos neonatais

Profissionais de saúde em contato direto com o paciente 
Do ponto de vista de saúde pública, considera-se que a efetividade (impacto após introdução na rotina) é mais relevante do que a eficácia avaliada através de estudos clínicos realizados em condições controladas (préregistro). A primeira informação sobre efetividade da Tdap recomendada para adolescentes vem de um estudo realizado em uma região do Canadá, onde essa vacina foi introduzida para escolares com idade entre 14 a 16 anos. A aceitação da vacina administrada em escolas foi excelente (cobertura 95\%) e a vacinação em massa levou a uma dramática redução na incidência da coqueluche tanto no grupo vacinado, como nos escolares não vacinados com idade entre 10 e 14 anos (84\% redução na incidência). Esse estudo demonstrou que a vacinação em larga escala é capaz de conferir proteção indireta (herd immunity) ${ }^{88}$.

A efetividade da vacina Tdap para adolescentes também foi demonstrada por meio de um estudo caso-controle, realizado na França, que demonstrou proteção de $80 \%$ em adolescentes vacinados há menos de seis anos ${ }^{89}$, assim como na recente epidemia registrada na Califórnia (Estados Unidos da América) ${ }^{93}$. Entretanto, durante uma epidemia de coqueluche constatada nas Ilhas Virgens (Grã-Bretanha) que acometeu $10 \%$ de 499 estudantes, a efetividade foi mais baixa $(65,6 \%)^{90}$. A efetividade depende da força de infecção, que por sua vez varia depende não apenas da imunidade individual, mas também da imunidade coletiva propiciada por altas coberturas vacinais.

A vacinação de todos os contatos próximos de bebês (incluindo familiares, cuidadores e profissionais de saúde) é conhecida como estratégia cocoon ou cocconing (casulo) e visa impedir que pessoas contaminadas pela coqueluche transmitam a bactéria aos lactentes com idade insuficiente para terem recebido o esquema de vacinação básico, formando uma barreira protetora ao redor do bebê. Essa estratégia vem sendo recomendada por diversas autoridades de saúde $7-11,12,14,20,21,23,24-28,34,35,40,42,46,50,51,54-65,70,85-87$. Embora muita ênfase seja dada à vacinação dos pais, em especial das mães, é fundamental lembrar que os irmãos também são fonte relevante de transmissão, principalmente quando não recebem as doses recomendadas no primeiro ano de vida e as doses de reforço ${ }^{60-65}$.

Estratégias de vacinação com foco em adolescentes e adultos, em geral, têm aceitação muito menor do que as voltadas para vacinação de lactentes. Oferecer as vacinas em escolas para os adolescentes e para os pais nas maternidades, de preferência sem custo para o paciente, e com estratégias que associam oferta gratuita da vacina com programas que envolvem a educação dos profissionais de saúde e da população-alvo da vacinação são as melhores formas de aumentar a aceitação das vacinas e as coberturas vacinais ${ }^{71,72,87}$.

A necessidade de vacinar grande número de indivíduos capazes de transmitir a pertussis aos bebês acarreta dificuldades enormes e dificulta a logística de vacinação. O grande número de mortes em bebês menores de dois meses, com idade insuficiente para receber qualquer dose de vacina contra coqueluche levou à busca de estratégias de vacinação que pudessem proteger a criança desde o momento do nascimento. A vacinação de gestantes com o intuito de conferir proteção ao bebê, através da transmissão de anticorpos por via transplacentária, é uma estratégia de sucesso na prevenção do tétano neonatal. Estima-se que, mesmo nos países em desenvolvimento, a maioria das gestantes tenha acesso às consultas de prénatal e que a excelente aceitação das vacinas contra tétano e, mais recentemente, contra influenza, deve facilitar a vacinação de gestantes contra a coqueluche ${ }^{81-85}$. Embora as vacinas Tdap e Tdap-IPV não tenham sido testadas em gestantes antes de seu registro, vacinas inativadas são consideradas seguras para uso em gestantes, quando há indicação, e desde seu licenciamento, as vacinas são indicadas pelas autoridades de saúde para gestantes em situação de risco, como epidemias ${ }^{4,17-21,23,66,67,84-86}$. Recomenda-se que a gestante receba a vacina contra pertussis após o primeiro trimestre de gestação para evitar possível associação casual com a ocorrência de aborto espontâneo. Embora se desconheçam os títulos de anticorpos protetores contra a coqueluche, estimase que os anticorpos transferidos da mãe para o bebê possam protegê-lo desde o nascimento, evitando o risco de coqueluche no período em que o bebê é mais vulnerável às complicações da doença. Estima-se que os anticorpos transmitidos da mãe para o bebê persistam elevados até 8 a 12 semanas de vida ${ }^{81-86}$. Estratégias que visam vacinar prioritariamente as gestantes são baseadas na maior viabilidade logística e econômica, assim como no fato de que quanto mais jovem o bebê, maior o risco de adquirir a doença da própria mãe. A administração de vacinas que não contêm agentes vivos é considerada segura para gestantes e pode beneficiar a mãe e o bebê. Tendo em vista essas considerações a as recentes epidemias de coqueluche registradas a partir de 2009, diversas autoridades de saúde têm recomendado a vacinação de mulheres grávidas ${ }^{4,20,21,84-86}$. Nos EUA, a vacina recomendada para gestantes é a Tdap ${ }^{84}$, enquanto no Reino Unido, foi indicado o uso da Tdap-IPV $75,76,85$.

Como essa orientação para uso das vacinas Tdap e Tdap-IPV não consta nas respectivas bulas, a indicação para uso em gestantes só pode ser feita a critério do médico assistente, quando existem orientações especificas das autoridades de saúde para sua utilização.

Um estudo recente confirmou que a maior passagem de anticorpos ocorre quando a gestante é vacinada no último trimestre de gestação e que, mesmo mulheres vacinadas previamente com Tdap podem ter baixos títulos de anticorpos ${ }^{93}$. Por esses motivos, o ACIP recomenda revacinar com Tdap mesmo as gestantes previamente vacinadas com essa vacina, de preferência, após 20 semanas de gestação e, pelo menos, 15 dias antes do parto $^{84}$. No Reino Unido, a vacina Tdap-IPV é indicada para gestantes entre 28 e 32 semanas de gestação ${ }^{86}$. 
Puérperas que não receberam a vacina durante a gestação, assim como os outros membros da família e cuidadores de lactentes jovens devem ser vacinados o mais rapidamente possível, de preferência, 15 dias antes de entrar em contato com o bebê ${ }^{84-86}$. Prematuros são de alto risco para complicações da coqueluche e, como a passagem de anticorpos só ocorre no último trimestre de gestação, mesmo que a mãe tenha sido vacinada, não estarão protegidos passivamente, sendo fundamental que pediatras e obstetras eduquem os familiares sobre a importância da estratégia cocoon e que também se vacinem, para evitar o risco de transmitir a coqueluche a esses bebês. O mesmo é válido para os cuidadores, como babás e profissionais que trabalham em berçários e creches ${ }^{19-24,73,87,96}$.

Finalmente, vale lembrar que as vacinas Tdap e Tdap-IPV podem ser administradas com intervalo mínimo de um mês após o uso da vacina Td ou Td-IPV e concomitantemente com outras vacinas contendo ou não agentes vivos, quando indicadas para adolescentes e adultos ${ }^{75,76,78,79,84}$.

Apesar das controvérsias sobre a efetividade e duração da proteção conferida por vacinas acelulares indicadas para crianças, adolescentes e adultos, a vacinação ainda é a forma mais efetiva de prevenir a doença ${ }^{9}$ Considerando-se que a proteção após vacinação é limitada, a revacinação de adolescentes e adultos deve ser implementada, visando não apenas sua proteção, mas também a proteção coletiva.

\section{CONCLUSÕES}

Pessoas de qualquer faixa etária podem transmitir a coqueluche aos lactentes, o grupo mais vulnerável às complicações e mortes. O subdiagnóstico é mais frequente em adolescentes e adultos que não apresentam sintomas característicos da doença. Quando a suspeita diagnóstica é feita após a terceira semana de tosse, o diagnóstico só pode ser confirmado por sorologia, um teste não disponível em nosso meio. As atuais estratégias de vacinação com foco em crianças são insuficientes para evitar as mortes em lactentes jovens. A proteção limitada conferida por vacinas e a confirmação de que adolescentes e adultos são fontes importantes de transmissão para da B. pertussis indicam a necessidade de novas estratégias de vacinação. Apesar das controvérsias sobre a efetividade de vacinas acelulares contra coqueluche, essas vacinas são mais seguras do que as vacinas de células inteiras, e são as únicas recomendadas para pessoas com mais de sete anos de idade e não há dúvida de que novas doses de reforço para adolescentes e adultos deverão ser implementadas. É necessário aprimorar a vigilância da pertussis em adolescentes e adultos para avaliar a duração da proteção conferida pelas vacinas, o intervalo ideal entre doses de reforço e para identificar as mais efetivas estratégias de vacinação. A educação da comunidade médica e leiga sobre os riscos da pertussis e os benefícios da vacinação de adolescentes e adultos é essencial para que as novas estratégias de vacinação sejam implantadas com sucesso. Enquanto não for possível oferecer doses de reforço para toda a população, é prioritário vacinar pessoas que têm contato com lactentes jovens, assim como manter elevadas coberturas vacinais em crianças no esquema básico e doses de reforço.

\section{REFERÊNCIAS}

1. World Health Organization (CH). Immunization surveillance, assessment and monitoring: Pertussis [Internet]. Geneve: World Health Organization; 2012 [updated 2012 Dec 6; cited 2012 Dec 12]. Available from: http://www.who.int/immunization_monitoring/ diseases/pertussis.

2. WHO Publication. Pertussis vaccines: WHO position paper-recommendations. Vaccine. 2011 Mar 16;29(13):2355-6. Available from: http://www.who.int/immunization/pertussis_grad_efficacy.pdf. doi: http://dx.doi.org/10.1016/j.vaccine.2010.11.059. Epub 2010 Dec 1. PubMed PMID: 21129396.

3. Centers for Disease Control and Prevention (US). Pertussis [Internet]. Atlanta: Centers for Disease Control and Prevention; 2012 [cited 2012 Dec 26]. Available from: http://www.cdc.gov/pertussis.

4. Ministério da Saúde (BR) (Departamento de Gestão e Incorporação de Tecnologias em S missão Nacional de Incorporação de Tecnologias no SUS). Vacina adsorvida difteria, tétano e pertussis (Acelular) - dTpa Para vacinação de gestantes [Internet]. Brasília: Ministério da Saúde; 2012 [acesso em 26 dez 2012]. 6 p. Relatório Nº. 46. Disponível em: http://portal.saude.gov.br/portal/arquivos/pdf/Relatorio_VacinadTPa _Gestantes_CP.pdf.

5. DATASUS, SINAN (Sistema de Informação de Agravos de Notificação) [base de dados na Internet]. Brasília: Ministério da Saúde. 2013 - Tabulação de dados: coqueluche; [acesso em 12 Abr 2013]. Disponível em: http:// dtr2004.saude.gov.br/sinanweb/tabnet/dh?sinan/coqueluche/ bases/ coquebr.def
6. DATASUS [base de dados na internet]. Brasília: Ministério da Saúde. 2012 - Morbidade hospitalar do SUS - por local de internação: Brasil; [acesso em 5 Nov 2012]. Disponível em: http://tabnet.datasus.gov.br/ cgi/deftohtm.exe?sih/cnv/miuf.def.

7. Centers for Disease Control and Prevention (US). Notifiable diseases and mortality tables. MMWR Morb Mortal Wkly Rep [Internet]. 2011 Jan 7 [cited 2012 Dec 26];59(52):1704-17. Available from: http:// www.cdc.gov/mmwr/preview/mmwrhtml/mm5952md.htm?s_ cid=mm5952md_w. Erratum for: MMWR Morb Mortal Wkly Rep. 2011 Mar 4;60(08);251.

8. Winter K, Harriman K, Zipprich J, Schechter R, Talarico J, Watt J, Chavez G. California pertussis epidemic, 2010. J Pediatr [Internet]. 2012 Dec [cited 2012 Dec 26];161(6):1091-6. Available from: http:// download.journals.elsevierhealth.com/pdfs/journals/0022-3476/ PIIS0022347612005586.pdf. doi: http://dx.doi.org/10.1016/j. jpeds.2012.05.041. Epub 2012 Jul 21. PubMed PMID: 22819634.

9. Centers for Disease Control and Prevention (US). Pertussis epidemic Washington, 2012. MMWR Morb Mortal Wkly Rep [Internet]. 2012 Jul 20 [cited 2012 Dec 26];61(28):517-22. Available from: http://www.cdc.gov/ mmwr/preview/mmwrhtml/mm6128a1.htm. PubMed PMID: 22810264.

10. Fisman DN, Tang P, Hauck T, Richardson S, Drews SJ, Low DE, Jamieson F. Pertussis resurgence in Toronto, Canada: a population-based study including test-incidence feedback modeling. BMC Public Health [Internet]. 2011 Sep 7 [cited 2012 Dec 26];11:694. Available from: 
http://www.biomedcentral.com/1471-2458/11/694. doi: http:// dx.doi.org/10.1186/1471-2458-11-694. PubMed PMID: 21899765; PubMed Central PMCID: PMC3189138.

11. Australian Government (Department of Health and Ageing). Pertussis [Internet]. Queensland: Australian Government; 2012 [updated 2013 Feb 26; cited 2012 Dec 26]. Available from: http://www.health.gov. au/internet/immunise/publishing.nsf/Content/immunise-pertussis.

12. Wall R, Bell A, Theobald J. Pertussis (whooping cough) epidemiology in Waikato, New Zealand: 2000-2009. N Z Med J. 2011 Apr 15;124(1332):52-61. PubMed PMID: 21747424.

13. European Centre for Disease Prevention and Control (SE). Pertussis [Internet]. Stockholme: ECDC; 2012 [cited 2012 Dec 26]. Available from: http://www.ecdc.europa.eu/en/activities/surveillance/euvac/ case_definition/pages/pertusis.aspx.

14. Public Health England (GB). Pertussis [Internet]. London: Public Health England; 2012 [cited 2012 Dec 26]. Available from: http://www.hpa. org.uk/Topics/InfectiousDiseases/InfectionsAZ/WhoopingCough/.

15. Sociedad Canaria de Pediatría Extrahospitalaria (ES). Epidemia de Tosferina [Internet]. Santa Cruz de Tenerife: Sociedad Canaria de Pediatría Extrahospitalaria; 2012 [accedido en 2012 Out 19]. Disponible en: http://www.sepexpal.org/2012/10/24/epidemia-detosferina-1-042-casos-declarados-78-ingresos-hospitalarios-14-enunidad-de-cuidados-intensivos-una-nina-menor-de-1-mes-fallecida/.

16. Zepp F, Heininger U, Mertsola J, Bernatowska E, Guiso N, Roord J, et al. Rationale for pertussis booster vaccination throughout life in Europe. Lancet Infect Dis. 2011 Jul;11(7):557-70. doi: http://dx.doi. org/10.1016/S1473-3099(11)70007-X. Epub 2011 May 18. Review. PubMed PMID: 21600850.

17. Ulloa-Gutierrez R, Avila-Aguero ML. Pertussis in Latin America: current situation and future vaccination challenges. Expert Rev Vaccines. 2008 Dec;7(10):1569-80. doi: http://dx.doi. org/10.1586/14760584.7.10.1569. Review. PubMed PMID: 19053212.

18. Nieto Guevara J, Luciani K, Montesdeoca Melián A, Mateos Durán M, Estripeaut D. Hospitalizaciones por Bordetella pertussis: experiencia del Hospital del Niño de Panamá, periodo 2001-2008. An Pediatr (Barc) [Internet]. 2010 Mar [cited 2012 Dec 26];72(3):172-8. Available from: http://apps.elsevier.es/watermark/ctl_servlet?_f=10\&pident_ articulo $=13148087 \&$ pident_usuario $=0 \&$ pcontactid $=\&$ pident revista $=37 \&$ ty=68\&accion=L\&origen=elsevier\&web=www.elsevier. es\&lan=es\&fichero=37v72n03a13148087pdf001.pdf. doi: http:// dx.doi.org/10.1016/j.anpedi.2009.11.012. Epub 2010 Feb 12. Spanish. PubMed PMID: 20153272.

19. Montevideo. Ministerio de Salud Pública (UY). Esquema de vacunación y nuevo plan de inoculación [Internet]. Decreto Interno №. 001-3-6826/2011, de 19 de dezembro de 2011 [acesso em 26 Dez 2012]. Recomendações do Comitê Nacional de Vacinação para as mudanças no calendário vacinação atual. Disponível em: http:// www.msp.gub.uy/uc_5971_1.html.

20. Pan American Health Organization (US). Epidemiological alert: pertussis (whooping cough) [Internet]. Washington: PAHO; 2012 Mar 2 [updated 2012 Mar 29; cited 2012 Dec 26]. Available from: http://new. paho.org/hq/index.php?option=com_content\&task= view\&id=6483\& $\mathrm{Itemid}=2291$.

21. Ministerio de Salud (AR). Tos convulsa: Aumento de casos y muertes. Alerta Epidemiológico [Internet]. 2012 Jan 25 [accedido $2012 \mathrm{Fev}$ 23];1:1-12. Disponible en: http://www.msal.gov.ar/images/stories/ alertas_epidemiologia/2012/alerta-1-tos-convulsa-2012.pdf.
22. Secretaria de Salud (CO). Respuesta a la enfermedad respiratoria aguda. Boletín Salud Respiratoria [Internet]. 2012 [acesso 2012 Maio 5];2(3):1-21. Disponible en: https://www.google. com.br/url?sa $=t \& r c t=j \& q=\&$ esrc $=s \&$ source $=$ web $\& c d=2 \& v e d=$ 0CDgQFjAB \&url=http\%3A\%2F\%2Fwww.saludcapital. gov.iones\%2FVigilancia\% 2520 en $\% 2520$ Salud $\%$ $2 \mathrm{FBoletines} \% 2520 \mathrm{~S}$ alud\% 2520 respiratoria\% 2520 Temporada\% 2520 invernal\%2 FBOLETIN \% 2520 SALUD\% 2520 RESPIRATORIA\% 2520 VOLUMEN\% $25202 \% 2520$ No.\%25203\%2520\%2520ABRIL\%252028\% 2520AL\%252005\%2520DE\%2520MAYO\%25202012.ppt\&ei=AOF2UZ qXBMWrOAGW04DICA\&usg=AFQjCNF85c6-eCeqY1gUe1MMPo5zVP K9Gg\&bvm=bv.45580626,d.dmQ

23. Potin M, Cerda J, Contreras L, Muñoz A, Ripoll E, Vergara R. [Modification of pertussis vaccination schedule in Chile, immunization of special groups and control strategies: Commentary from the Consultive Committee of Immunizations of The Chilean Society of Infectious Diseases]. Rev Chilena Infectol [Internet]. 2012 Jun [cited 2012 Dec 26];29(3):307-11. Available from: http://www.scielo.cl/ scielo.php?script=sci_arttext\&pid=S0716-10182012000300008\&/n g=en\&nrm=iso\&tlng=en. doi: http://dx.doi.org/10.1590/S071610182012000300008. Spanish. PubMed PMID: 23096469.

24. Edwards K, Decker M. Pertussis vaccines. In: Plotkin S, Orenstein W, editors. Vaccines. 6th ed. Philadelphia: Saunders, 2008. p. 467-517.

25. Forsyth $\mathrm{KD}$, Wirsing von Konig $\mathrm{CH}$, Tan $\mathrm{T}$, Caro J, Plotkin S. Prevention of pertussis: recommendations derived from the second Global Pertussis Initiative roundtable meeting. Vaccine. 2007 Mar 30;25(14):2634-42. Epub 2006 Dec 22. PubMed PMID: 17280745.

26. Guiso N. Bordetella pertussis and pertussis vaccines. Clin Infect Dis [Intenet]. 2009 Nov [cited 2012 Set 10];49(10):1565-9. Available from: http://cid.oxfordjournals.org/content/49/10/1565.full.pdf+html. doi: http://dx.doi.org/10.1086/644733.

27. Wendelboe AM, Van Rie A, Salmaso S, Englund JA. Duration of immunity against pertussis after natural infection or vaccination. Pediatr Infect Dis J. 2005 May;24(5 Suppl):S58-61. Review. PubMed PMID: 158769927.

28. Edwards KM. Overview of pertussis: focus on epidemiology, sources of infection, and long term protection after infant vaccination. Pediatr Infect Dis J. 2005 Jun;24(6 Suppl):S104-8. PubMed PMID: 15931137.

29. Bisgard KM, Pascual FB, Ehresmann KR, Miller CA, Cianfrini C, Jennings CE, et al. Infant pertussis: who was the source? Pediatr Infect Dis J. 2004 Nov;23(11):985-9. PubMed PMID: 15545851.

30. Schellekens J, von Konig $\mathrm{CH}$, Gardner P. Pertussis sources of infection and routes of transmission in the vaccination era. Pediatr Infect Dis J. 2005 May;24(5 Suppl): S19-24. Review. PubMed PMID: 15876919.

31. Wiley KE, Zuo Y, Macartney KK, McIntyre PB. Sources of pertussis infection in young infants: A review of key evidence informing targeting of the cocoon strategy. Vaccine. $2013 \operatorname{Jan} 11 ; 31(4): 618-25$. doi: http://dx.doi.org/10.1016/j.vaccine.2012.11.052. Epub 2012 Nov 29. PubMed PMID: 23200883.

32. Haberling DL, Holman RC, Paddock CD, Murphy TV. Infant and maternal risk factors for pertussis-related infant mortality in the United States, 1999 to 2004. Pediatr Infect Dis J. 2009 Mar;28(3):1948. doi: http://dx.doi.org/10.1097/INF.0b013e31818c9032. PubMed PMID: 19209089

33. Bonmarin I, Poujol I, Levy-Bruhl D. Nosocomial infections and community clusters of pertussis in France, 2000-2005. Euro Surveill. 2007 Nov 1;12(11):E11-2. PubMed PMID: 18005652. 
34. Centro de Vigilância Epidemiológica Prof. Alexandre Vranjac (BR). Coqueluche: Distribuição de casos confirmados, óbitos, coeficiente de incidência e letalidade, segundo ano de início de sintomas e faixa etária, Estado de São Paulo, 2000 a 2012 [Internet]. São Paulo: Centro de Vigilância Epidemiológica Prof. Alexandre Vranjac; 2012 [acesso 2012 Dez 26]. Disponível em: http://www.cve.saude.sp.gov.br/htm/ resp/coque_dados.html.

35. Leite D, Blanco RM, Melo LCV, Vaz TMI, Carvalhanas TRMP, Yu ALF et al. Comparative analysis of 3,961 nasopharyngeal samples for pertussis diagnosis using RT-PCR and culture in 2010/2011, São Paulo - Brazil [abstract]. In: 30th Annual Meeting of the European Society of Paediatric Infectious Diseases; 2012; Thessaloniki. Greece: European Society of Paediatric Infectious Diseases; 2012.

36. Korppi M, Hiltunen J. Pertussis is common in nonvaccinated infants hospitalized for respiratory syncytial virus infection. Pediatr Infect Dis J. 2007 Apr;26(4):316-8. PubMed PMID: 17414394.

37. Nuolivirta K, Koponen $\mathrm{P}, \mathrm{He} \mathrm{Q}$, Halkosalo A, Korppi $\mathrm{M}$, Vesikari T, et al. Bordetella pertussis infection is common in nonvaccinated infants admitted for bronchiolitis. Pediatr Infect Dis J. 2010 Nov;29(11):10135. PubMed PMID: 21046700.

38. Zouari A, Touati A, Smaoui H, Brun D, Kasdaghli K, Menif K et al. Dual infection with Bordetella pertussis and Mycoplasma pneumoniae in three infants: case reports. Infection. 2012 Apr;40(2):213-7. doi: http://dx.doi.org/10.1007/s15010-011-0179-4. Epub 2011 Aug 23. PubMed PMID: 21861122.

39. Campins-Martí M, Cheng HK, Forsyth K, Guiso N, Halperin S, Huang LM. Recommendations are needed for adolescent and adult pertussis immunisation: rationale and strategies for consideration. Vaccine. 2001 Dec 12;20(5-6):641-6. Review. PubMed PMID: 11738728.

40. Centers for Disease Control and Prevention (US). Local health department costs associated with response to a school-based pertussis outbreak --- Omaha, Nebraska, September-November 2008. MMWR Morb Mortal Wkly Rep [Internet]. 2011 Jan 14 [cited 2012 Dec 26];60(1):5-9. Available from: http://www.cdc.gov/mmwr/ preview/mmwrhtml/mm6001a2.htm. PubMed PMID: 21228761.

41. Senzilet LD, Halperin SA, Spika JS, Alagaratnam M, Morris A, Smith B. Sentinel Health Unit Surveillance System Pertussis Working Group. Pertussis is a frequent cause of prolonged cough illness in adults and adolescents. Clin Infect Dis [Internet]. 2001 Jun 15 [cited 2012 Dec 26];32(12):1691-7. Available from: http://cid.oxfordjournals.org/content/32/12/1691.full. pdf+html. Epub 2001 May 21. PubMed PMID: 11360208.

42. Wymann MN, Richard JL, Vidondo B, Heininger U. Prospective pertussis surveillance in Switzerland, 1991-2006. Vaccine. 2011 Mar 3;29(11):2058-65. doi: http://dx.doi.org/10.1016/j. vaccine.2011.01.017. Epub 2011 Jan 18. PubMed PMID: 21251904.

43. Bonhoeffer J, Bär $G$, Riffelmann $M$, Solèr $M$, Heininger $U$. The role of Bordetella infections in patients with acute exacerbation of chronic bronchitis. Infection. 2005 Feb;33(1):13-7. PubMed PMID: 15750754.

44. Capili CR, Hettinger A, Rigelman-Hedberg N, Fink L, Boyce T, Lahr B, et al. Increased risk of pertussis in patients with asthma. J Allergy Clin Immunol [Internet]. 2012 Apr [cited 2012 Dec 26];129(4):957-63. Available from: http://www.ncbi.nlm.nih.gov/ pmc/articles/PMC3321509/pdf/nihms363891.pdf. doi: http://dx.doi. org/10.1016/j.jaci.2011.11.020. Epub 2011 Dec 28. PubMed PMID: 22206778; PubMed Central PMCID: PMC3321509.

45. Burgess JA, Abramson MJ, Gurrin LC, Byrnes GB, Matheson MC, Wharton $\mathrm{CL}$, et al. Childhood infections and the risk of asthma: a longitudinal study over 37 years. Chest. 2012 Sep;142(3):647-54. PubMed PMID: 22459783.
46. Liu BC, McIntyre P, Kaldor JM, Quinn HE, Ridda I, Banks E. Pertussis in older adults: prospective study of risk factors and morbidity. Clin Infect Dis. 2012 Dec;55(11):1450-6. doi: http://dx.doi.org/10.1093/ cid/cis627. Epub 2012 Jul 17. PubMed PMID: 22806592.

47. Tatti KM, Tondella ML. Utilization of multiple real-time PCR assays for the diagnosis of Bordetella spp. in clinical specimens. Methods Mol Biol. 2013;943:135-47. doi: http://dx.doi.org/10.1007/978-1-60327353-4_9. PubMed PMID: 23104287.

48. Cattaneo LA, Reed GW, Haase DH, Wills MJ, Edwards KM. The seroepidemiology of Bordetella pertussis infections: a study of persons ages 1-65 years. J Infect Dis. 1996 May;173(5):1256-9. PubMed PMID: 8627081.

49. Kretzschmar M, Teunis PF, Pebody RG. Incidence and reproduction numbers of pertussis: estimates from serological and social contact data in five European countries. PLoS Med [Internet]. 2010 Jun 22 [cited 2012 Dec 26];7(6):e1000291. Available from: http://www.plosmedicine.org/article/info\%3Adoi\%2F10.1371\% 2Fjournal.pmed.1000291. doi: http://dx.doi.org/10.1371/journal. pmed.1000291. PubMed PMID: 20585374; PubMed Central PMCID: PMC2889930.

50. Miyashita N, Kawai Y, Yamagucho T, Ouhci K, Kurose K, Oka M. Outbreak of pertussis in a university laboratory. Intern Med [Internet]. 2011 [cited 2012 Dec 26];50(8):879-85. Available from: https:// www.jstage.jst.go.jp/article/internalmedicine/50/8/50_8_879/_pdf. doi: http://dx.doi.org/10.2169/internalmedicine.50.4768. Joi: JST. JSTAGE/internalmedicine.50.4768. Epub 2011 Apr 15. PubMed PMID: 21498936.

51. Lai FY, Thoon KC, Ang LW, Tey SH, Heng D, Cutter J, et al. Comparative seroepidemiology of pertussis, diphtheria and poliovirus antibodies in Singapore: Warning pertussis immunity in a highly immunized population and the need for adolescent booster doses. Vaccine. 2012 May 21;30(24):3566-71. doi: http://dx.doi.org/10.1016/j. vaccine.2012.03.059. Epub 2012 Apr 1. PubMed PMID: 22475863.

52. He Q, Barkoff AM, Mertsola J, Glismann S, Bacci S; European Bordetella expert group (EUpertstrain); European surveillance network for vaccine-preventable diseases (EUVAC.NET). High heterogeneity in methods used for the laboratory confirmation of pertussis diagnosis among European countries, 2010: integration of epidemiological and laboratory surveillance must include standardisation of methodologies and quality assurance. Euro Surveill [Internet]. 2012 Aug 9 [cited 2012 Dec 26];17(32). Available from: http://www. eurosurveillance.org/images/dynamic/EE/V17N32/art20239.pdf. doi: http://dx.doi.org/pii:20239. PubMed PMID: 22913939.

53. Pawloski LC, Kirkland KB, Baughman AL, Martin MD, Talbot EA, Messonnier NE et al. Does Tdap Vaccination Interfere with Serodiagnosis of Pertussis Infection? Clin Vaccine Immunol [Internet]. 2012 Jun [cited $2012 \mathrm{Dec} 26] ; 19(6): 875-80$. Available from: http://cvi.asm.org/content/19/6/875.full.pdf+html. doi: http:// dx.doi.org/10.1128/CVI.05686-11. Epub 2012 Apr 25. PubMed PMID: 22539469; PubMed Central PMCID: PMC3370450.

54. Elliott E, McIntyre P, Ridley G, Morris A, Massie J, McEniery J, Knight G. National study of infants hospitalized with pertussis in the acellular vaccine era. Pediatr Infect Dis J. 2004 Mar;23(3):246-52. PubMed PMID: 15014301.

55. Wendelboe AM, Hudgens MG, Poole C, Van Rie A. Estimating the role of casual contact from the community in transmission of Bordetella pertussis to young infants. Emerg Themes Epidemiol [Internet]. 2007 Oct 19 [cited 2012 Dec 26];4:15. Available from: http://www. ete-online.com/content/pdf/1742-7622-4-15.pdf. PubMed PMID: 17949498; PubMed Central PMCID: PMC2170437. 
56. Wendelboe AM, Njamkepo E, Bourillon A, Floret DD, Gaudelus J, Gerber M et al. Transmission of Bordetella pertussis to young infants. Pediatr Infect Dis J. 2007 Apr;26(4):293-9. PubMed PMID: 17414390.

57. Craig AS, Wright SW, Edwards KM, Greene JW, Haynes M, Dake AD, Schaffner W. Outbreak of pertussis on a college campus. Am J Med. 2007 Apr;120(4):364-8. PubMed PMID: 17398232.

58. Kowalzik F, Barbosa AP, Fernandes VR, Carvalho PR, Avila-Aguero ML, Goh DY, et al. Prospective multinational study of pertussis infection in hospitalized infants and their household contacts. Pediatr Infect Dis J. 2007 Mar;26(3):238-42. PubMed PMID: 17484221.

59. Chuk LM, Lambert SB, May ML, Beard FH, Sloots TP, Selvey CE et al. Pertussis in infants: how to protect the vulnerable? Commun Dis Intell Q Rep. 2008 Dec;32(4):449-56. PubMed PMID: 19374274.

60. Baptista PN, Magalhães VS, Rodrigues LC. Children with pertussis inform the investigation of other pertussis cases among contacts. BMC Pediatr [Internet]. 2007 May 22 [cited 2012 Dec 26];7:21. Available from: http://www.biomedcentral.com/content/pdf/1471-2431-7-21. pdf. PubMed PMID: 17518997; PubMed Central PMCID: PMC1894795.

61. Baptista PN, Magalhães VS, Rodrigues LC. The role of adults in household outbreaks of pertussis. Int J Infect Dis. 2010 Feb;14(2):e111-4. doi: http://dx.doi.org/10.1016/j.ijid.2009.03.026. Epub 2009 Jun 25. PubMed PMID: 19559636.

62. de Greeff SC, Mooi FR, Westerhof A, Verbakel JM, Peeters MF, Heuvelman $\mathrm{CJ}$ et al. Pertussis disease burden in the household: how to protect young infants. Clin Infect Dis [Internet]. 2010 May 15 [cited 2012 Dec 26];50(10):1339-45. Available from: http://cid. oxfordjournals.org/content/50/10/1339.full.pdf+html. doi: http:// dx.doi.org/10.1086/652281. PubMed PMID: 20370464.

63. Jardine $\mathrm{A}$, Conaty $\mathrm{SJ}$, Lowbridge $\mathrm{C}$, Staff $\mathrm{M}$, Vally H. Who gives pertussis to infants? Source of infection for laboratory confirmed cases less than 12 months of age during an epidemic, Sydney, 2009. Commun Dis Intell. 2010 Jun;34(2):116-21. PubMed PMID: 20677421.

64. Perret C, Viviani T, Peña A, Abarca K, Ferrés M. Fuentes de infección de Bordetella pertussis en lactantes hospitalizados por coqueluche. Rev Med Chil [Internet]. 2011 Apr [cited 2012 Dec 26];139(4):44854. Available from: http://www.scielo.cl/scielo.php?script=sci arttext\&pid=S0034-98872011000400005\&tlng=en\&lng=en\&nrm =iso. doi: http://dx.doi.org/10.4067/S0034-98872011000400005. PubMed PMID: 21879182.

65. de Greeff SC, de Melker HE, Westerhof A, Schellekens JF, Mooi $F R$, van Boven M. Estimation of household transmission rates of pertussis and the effect of cocooning vaccination strategies on infant pertussis. Epidemiology. 2012 Nov;23(6):852-60. doi: http://dx.doi. org/10.1097/EDE.0b013e31826c2b9e. PubMed PMID: 23018969.

66. Kretsinger K, Broder KR, Cortese MM, Joyce MP, Ortega-Sanchez I, Lee GM et al. Preventing tetanus, diphtheria, and pertussis among adults: use of tetanus toxoid, reduced diphtheria toxoid and acellular pertussis vaccine recommendations of the Advisory Committee on Immunization Practices (ACIP) and recommendation of ACIP, supported by the Healthcare Infection Control Practices Advisory Committee (HICPAC), for use of Tdap among health-care personnel. MMWR Recomm Rep [Internet]. 2006 [cited 2012 Dec 26];55(RR17):1-37. Available from: http://www.cdc.gov/mmwr/preview/ mmwrhtml/rr5517a1.htm. PubMed PMID: 17167397.

67. Murphy TV, Slade BA, Broder KR, Kretsinger K, Tiwari T, Joyce PM, et al. Prevention of pertussis, tetanus, and diphtheria among pregnant and postpartum women and their infants recommendations of the Advisory Committee on Immunization Practices (ACIP). MMWR Recomm Rep [Internet]. 2008 May 30 [cited 2012 Dec 26];57(RR-
4):1-51. Available from: http://www.cdc.gov/mmwr/preview/ mmwrhtml/rr5704a1.htm. Review. Erratum in: MMWR Morb Mortal Wkly Rep. 2008 Jul 4;57(26):723. PubMed PMID: 18509304.

68. Coudeville L, Van Rie A, Getsios D, Caro JJ, Crépey P, Nguyen VH. Adult vaccination strategies for the control of pertussis in the United States: an economic evaluation including the dynamic population effects. PLoS One [Internet]. 2009 Jul 16 [cited 2012 Dec 26];4(7):e6284. Available from: http://www.plosone.org/ article/info\%3Adoi\%2F10.1371\%2Fjournal.pone.0006284. doi: http://dx.doi.org/10.1371/journal.pone.0006284. PubMed PMID: 19606227; PubMed Central PMCID: PMC2707617.

69. McCormack PL. Reduced-antigen, combined diphtheria, tetanus and acellular pertussis vaccine, adsorbed (Boostrix ${ }^{\circledR}$ ): a review of its properties and use as a single-dose booster immunization. Drugs. 2012 Sep 10;72(13):1765-91. doi: http://dx.doi.org/10.2165/11209630000000000-00000. Review. PubMed PMID: 22931522.

70. Siegrist CA. [New Swiss recommendations for adult boosters against pertussis, tetanus and diphtheria]. Rev Med Suisse. 2012 Jan 18;8(324):125-8. [French]. PubMed PMID: 23185823.

71. Tan TQ, Gerbie MV. Pertussis and patient safety: implementing Tdap vaccine recommendations in hospitals. Jt Comm J Qual Patient Saf. 2010;36(4):173-8. PubMed PMID: 20402374.

72. Healy CM, Rench MA, Baker CJ. Implementation of cocooning against pertussis in a high-risk population. Clin Infect Dis [Internet]. 2011 Jan 15[cited 2012 Dec 26];52(2):157-62. Available from: http://cid. oxfordjournals.org/content/52/2/157.full.pdf+html. doi: http:// dx.doi.org/10.1093/cid/ciq001. PubMed PMID: 21288837.

73. Maltezou HC, Wicker S, Borg M, Heininger U, Puro V, Theodoridou $M$,.et al. Vaccination policies for health-care workers in acute healthcare facilities in Europe. Vaccine. 2011 Nov 28;29(51):9557-62. doi: http://dx.doi.org/10.1016/j.vaccine.2011.09.076. Epub 2011 Sep 29. PubMed PMID: 21964058.

74. European Centre for Disease Prevention and Control (SE) [Internet]. Pertussis vaccination overview in European countries. Sweden: European Centre for Disease Prevention and Control; 2012 [cited 2012 Dec 26]. Available from: http://ecdc.europa.eu/en/activities/ surveillance/euvac/schedules/pages/pertussis_schedule.aspx.

75. Larnaudie S Guiso N, Baptiste C, Desaint C, Desforges L, Lebon Pet al.Humoral immunity of dTap-IPV vaccine (REPEVAX ${ }^{\circledR}$ ) administered one month after dT-IPV vaccine (REVAXIS ${ }^{\circledR}$ ) in adults with unknown vaccination history. Hum Vaccin. 2010 Oct;6(10):829-34. doi: http://dx.doi. org/10.4161/hv.6.10.12582. Epub 2010 Oct 1. PubMed PMID: 20864810.

76. Beytout J, Launay O, Guiso N, Fiquet A, Baudin M, Richard P et al. Safety of Tdap-IPV given one month after Td-IPV booster in healthy young adults: a placebo-controlled trial. Hum Vaccin. 2009 May;5(5):315-21. Epub 2009 May 1. PubMed PMID: 19011374.

77. Vesikari T, Van Damme P, Lindblad N, Pfletschinger U, Radley D, Ryan $D$, et al. An open-label, randomized, multicenter study of the safety, tolerability, and immunogenicity of quadrivalent human papillomavirus (types 6/11/16/18) vaccine given concomitantly with diphtheria, tetanus, pertussis, and poliomyelitis vaccine in healthy adolescents 11 to 17 years of age. Pediatr Infect Dis J. 2010 Apr;29(4):314-8. doi: http:// dx.doi.org/10.1097/INF.0b013e3181c177fb. PubMed PMID : 19952980.

78. Scott LJ. Spotlight on $\operatorname{Tdap}_{5}$ vaccine $\left(\right.$ Covaxis $^{\circledR}$ ) as a single-booster immunization for the prevention of tetanus, diphtheria, and pertussis: in children (aged $\geq 4$ years), adolescents, and adults. Paediatr Drugs. 2011 Apr 1;13(2):133-5. doi: http://dx.doi.org/10.2165/11206990000000000-00000. PubMed PMID: 21351814. 
79. Laurichesse H, Zimmermann U, Galtier F, Launay O, Duval X, Richard $P$, Sadorge C, Soubeyrand B. Immunogenicity and safety results from a randomized multicenter trial comparing a Tdap-IPV vaccine (REPEVAX $\left({ }^{\circledR}\right)$ ) and a tetanus monovalent vaccine in healthy adults: New considerations for the management of patients with tetanusprone injuries. Hum Vaccin Immunother. 2012 Oct 2;8(12). [Epub ahead of print]. PubMed PMID: 23032160.

80. Prevots DR, Burr RK, Sutter RW, Murphy TV; Advisory Committee on Immunization Practices (ACIP). Poliomyelitis prevention in the United States. MMWR Recomm Rep [Internet]. 2000 May 9 [cited 2012 Dec 26];49(RR-5):1-22; quis CE1-7. Available from: http://www.cdc.gov/ mmwr/preview/mmwrhtml/rr4905a1.htm. PubMed PMID: 15580728.

81. Halperin BA, Halperin SA. The reemergence of pertussis and infant deaths: is it time to immunize pregnant women? Future Microbiol [Internet]. 2011 Apr [cited 2012 Dec 26];6(4):367-9. Available from: http://www.futuremedicine.com/doi/pdf/10.2217/fmb.11.15. doi: http://dx.doi.org/10.2217/fmb.11.15. PubMed PMID: 21526936.

82. Halperin BA, Morris A, Mackinnon-Cameron D, Mutch J, Langley $J M$, McNeil SA et al. Kinetics of the antibody response to tetanusdiphtheria-acellular pertussis vaccine in women of childbearing age and postpartum women. Clin Infect Dis [Internet]. 2011 [cited 2012 Dec 26];53(9):885-92. Available from: http://cid.oxfordjournals.org/ content/53/9/885.full.pdf+html. doi: http://dx.doi.org/10.1093/cid/ cir538. Epub 2011 Sep 26. PubMed PMID: 21946190.

83. Peters TR, Banks GC, Snively BM, Poehling KA. Potential impact of parental Tdap immunization on infant pertussis hospitalizations. Vaccine. 2012 Aug 10;30(37):5527-32. doi: http://dx.doi. org/10.1016/j.vaccine.2012.06.047. Epub 2012 Jun 27. PubMed PMID: 22749592; PubMed Central PMCID: PMC3407342.

84. Centers for Disease Control and Prevention (US). Updated recommendations for use of tetanus toxoid, reduced diphtheria toxoid and acellular pertussis vaccine (Tdap) in pregnant women and persons who have or anticipate having close contact with an infant aged $<12$ months --- Advisory Committee on Immunization Practices (ACIP), 2011. MMWR Morb Mortal Wkly Rep [Internet]. 2011 Oct 21[cited 2012 Dec 26];60(41):1424-6. Available from: http://www.cdc.gov/mmwr/ preview/mmwrhtml/mm6041a4.htm. PubMed PMID: 22012116.

85. Healy CM, Rench MA, Baker CJ. Importance of Timing of Maternal Combined Tetanus, Diphtheria, and Acellular Pertussis (Tdap) Immunization and Protection of Young Infants Clin Infect Dis. 2013 Feb;56(4):539-44. doi: http://dx.doi.org/10.1093/cid/cis923. Epub 2012 Oct 24. PubMed PMID: 23097585.

86. Billingsley M. Pregnant women in UK are offered whooping cough vaccine to protect newborns. Vaccine [Internet]. 2012 Nov 6 [cited 2012 Dec 26];30(48):6745-52. Available from: http://www.bmj. com/content/345/bmj.e6594.pdf\%2Bhtml . doi: http://dx.doi. org/.10.1136/bmj.e6594. PubMed PMID: 23045350.

87. Camenga DR, Kyanko K, Stepczynski J, Flaherty-Hewitt M, Curry L, Sewell $D$, et al. Increasing adult Tdap vaccination rates by vaccinating infant caregivers in the pediatric office. Acad Pediatr. 2012 JanFeb;12(1):20-5. doi: http://dx.doi.org/10.1016/j.acap.2011.11.003. PubMed PMID: 22243708.

88. Greenberg DP, Doemland M, Bettinger JA, Scheifele DW, Halperin SA, Waters V, et al. Epidemiology of pertussis and Haemophilus influenzae type $b$ disease in Canada with exclusive use of $a$ diphtheria-tetanus-acellular pertussis-inactivated poliovirusHaemophilus influenzae type $b$ pediatric combination vaccine and an adolescent-adult tetanus-diphtheria-acellular pertussis vaccine: implications for disease prevention in the United States. Pediatr Infect Dis J. 2009 Jun;28(6):521-8. doi: http://dx.doi.org/10.1097/ INF.0b013e318199d2fc. PubMed PMID: 19436236.

89. Berger F, Njamkepo E, Minaberry S, Mayet A, Haus-Cheymol R, Verret $C$, et al. Investigation on a pertussis outbreak in a military school: risk factors and approach to vaccine efficacy. Vaccine. 2010 Jul 19;28(32):5147-52. doi: http://dx.doi.org/10.1016/j. vaccine.2010.05.070. Epub 2010 Jun 9. Review. PubMed PMID: 20541009.

90. Wei SC, Tatti K, Cushing K, Rosen J, Brown K, Cassiday P et al. Effectiveness of adolescent and adult tetanus, reduced-dose diphtheria, and acellular pertussis vaccine against pertussis. Clin Infect Dis [Internet]. 2010 Aug 1 [cited 2012 Dec 26];51(3):315-21. Available from: http://cid.oxfordjournals.org/content/51/3/315.full. pdf+html. doi: http://dx.doi.org/10.1086/653938. PubMed PMID: 20578875.

91. Witt MA, Katz PH, Witt DJ. Unexpectedly limited durability of immunity following acellular pertussis vaccination in preadolescents in a North American outbreak. Clin Infect Dis. 2012 Jun;54(12):17305. doi: http://dx.doi.org/10.1093/cid/cis287. Epub 2012 Mar 15. PubMed PMID: 22423127

92. Klein NP, Bartlett J, Rowhani-Rahbar A, Fireman B, Baxter R. Waning protection after fifth dose of acellular pertussis vaccine in children. N Engl J Med [Internet]. 2012 Sep 13 [cited 2012 Dec 26];367(11): 1012-9. Available from: http://www.nejm.org/doi/pdf/10.1056/ NEJMoa1200850. doi: http://dx.doi.org/10.1056/NEJMoa1200850. PubMed PMID: 22970945.

93. Misegades LK, Winter K, Harriman K, Talarico J, Messonnier NE, Clark TA, Martin SW. Association of childhood pertussis with receipt of 5 doses of pertussis vaccine by time since last vaccine dose, California, 2010. JAMA [Internet]. 2012 Nov 28 [cited 2012 Dec 26];308(20):2126-32. Available from: http://jama.jamanetwork.com/ article.aspx?articleid=1456072. doi: http://dx.doi.org/10.1001/jama. 2012.14939. PubMed PMID: 23188029.

94. Helms C, Leask J, Tobbins SC, Chow MY, McIntyre P. Implementation of mandatory immunisation of ealthcare workers: observations from New South Wales, Australia. Vaccine 2011 Apr 5;29(16):2895-901. doi: http://dx.doi.org/10.1016/j.vaccine.2011.02.011. Epub 2011 Feb 21. PubMed PMID: 21338677.

95. Lindley MC, Lorick SA, Spinner JR, Mootrey GT, Ahmed F et al. Student vaccintion requirements of US health professional schools: a survey. Ann Intern Med. 2011 Mar 15;154(6):391-400. doi: http://dx.doi. org/10.1059/0003-4819-154-6-201103150-00004. PubMed PMID: 21403075.

96. Hope K, Butler M, Masey PD, Cashman P, Durreheim DN, Stephenson $J$ et al. Pertussis vaccination in child care workers: room for improvement in coverage, police and practice. BMC Pediatr [Internet].2012 Jul 13 [cited 2012 Dec 26]; 12:98. Available from: http://www.biomedcentral.com/content/pdf/1471-2431-12-98. pdf. doi: http://dx.doi.org/10.1186/147-241-12-98. PubMed PMID: 22794120; PubMed Central PMCID: PMC3411446.

Como citar este artigo / How to cite this article:

Bricks LF. Pertussis: novas estratégias de vacinação para prevenção de uma antiga doença. J Health Biol Sci. 2013 Abr-Jun;1(2):73-83. 